\title{
Probabilistic Analysis of a Ceramic Tile Production System Considering Various Redundant Subsystems and Inspection
}

\author{
Reena*, Rajeev Kumar \\ Department of Mathematics, M.D. University, Rohtak (Haryana), India \\ *Corresponding author: phogat.reena026@gmail.com \\ Received January 10, 2019; Revised February 19, 2019; Accepted February 28, 2019
}

\begin{abstract}
The present paper gives analysis of a stochastic model for a ceramic tile production system considering its five main subsystems viz. Ball Mill, Spray Dryer, Hydraulic Press, Glaze Line and Kiln and a storage system, Silo. It was considered that the failure of Ball Mill and Spray Dryer subsystems depends upon Silo while failure of other subsystems are independent of Silo. Also the storage in Silo is consumed during the repair/replacement of the failed subsystems then the system goes to failure otherwise it is operative. During the occurrence of failure in subsystems Hydraulic Press, Glaze Line and Kiln, leads to the failure in other subsequent subsystem and the repairman first inspects in which redundant unit of the subsystem has a fault and then accordingly repair/replacement is carried out. The system has been analysed using Markov process and regenerative point techniques. Various conclusions for the system regarding its reliability and availability are drawn by plotting several graphs for a particular case.
\end{abstract}

Keywords: ceramic tile production system, stochastic model, reliability, availability, Markov process and regenerative point technique

Cite This Article: Reena, and Rajeev Kumar, "Probabilistic Analysis of a Ceramic Tile Production System Considering Various Redundant Subsystems and Inspection." American Journal of Applied Mathematics and Statistics, vol. 7, no. 2 (2019): 79-85. doi: 10.12691/ajams-7-2-5.

\section{Introduction}

Just from the beginning of the inception of technological systems, over a long period of time anticipation of the public of the society has been that the systems to perform its work satisfactorily and accurately. To meet the challenge and expectation of the public, various reliability models have been elaborated since long by sevaral researchers taking into account different circumstances/aspects and acquiring system performance influencing measures. Kulshreshtha [1] investigated a multi-component system, EL-Sherbeny [2] analysed two-stage repair time system, Yusuf and Yusuf [3] determined the problem of three types of failures, Taneja and Sachdeva [4] discussed on optical communication process and Rajesh et al. [5] studied about the reliability of a gas turbine. All the above studies achieved different types of failures, which optimize the reliability of the systems.

Proschan [6] introduced redundancy of the system, Arora [7] investigated reliability of many standby redundant systems with priority, Dhillon [8] studied a multistate component redundant system having common cause failures. Wang et al. [9] determined the comparison of availability between four systems having warm standby subsystems with standby switching failures. Recently,
Reena and Kumar [10] obtained reliability analysis of a ceramic tile manufacture system having various subsystem failures. So, lot of studies has been done by several researchers taking redundant systems. In this paper we deal with a stochastic models developed for a ceramic tile production system considering its five main subsystems viz. Ball Mill, Spray Dryer, Hydraulic Press, Glaze Line and Kiln and a storage system, Silo that is used for storing raw material on the basis of information made while visiting the system. It was observed that the failure of Ball Mill and Spray Dryer subsystems depends upon Silo while failures of other subsystems are independent of Silo. If the storage in Silo is consumed during the repair/replacement of the failed subsystems then the system goes to failure otherwise it is operative. On failure of the system, subsystems Hydraulic Press, Glaze Line and Kiln are inspected by the repairman to judge in which subsystems Hydraulic Press, Glaze Line and Kiln fault has occurred and accordingly repair the subsystem. During the occurrence of fault in the subsystems Hydraulic Press, Glaze Line and Kiln leads to the failure in other subsequent subsystem.

\section{Assumption}

1. Subsystems Hydraulic Press, Glaze Line and Kiln having redundant subsystems. 
2. Inspection after each failure in redundant subsystem Hydraulic Press, Glaze Line and Kiln.

3. Failure in subsystems are independent.

4. There is a single repair facility that carries out repairs in FCFS pattern.

5. The system is as good as new after each repair.

6. All the random variables taken here are assumed mutually independent.

7. Switching is impeccable and instantaneous.

8. The failure time distributions are taken as exponential although the other time distributions assumed to be general.

\section{Notations}

$\lambda_{1} / \lambda_{2} / \lambda_{3} / \lambda_{4} / \lambda_{5}$ constant failure rate of $\mathrm{A} / \mathrm{B} / \mathrm{C} / \mathrm{D} / \mathrm{E}$ subsystems of the system

$\mathrm{X} \quad$ dust storage level at a time

$\mathrm{x}_{0} \quad$ required level of dust for the operation of the system

t* time duration in which dust storage reduced to required level $\mathrm{x} 0$

$\mathrm{p}_{1} \quad$ probability that the dust storage (capacity) is more than the required level $=\mathrm{P}(\mathrm{X} \geq \mathrm{x} 0)$

$\mathrm{q}_{1} \quad$ probability that the dust storage (capacity) is less than the required level $=\mathrm{P}(\mathrm{X}<\mathrm{x} 0)$

$\mathrm{p}_{2} \quad$ probability that the repair is done before the dust storage (capacity) goes less than required level $\mathrm{x} 0$

$\mathrm{q}_{2}$ probability that the repair is done after the dust storage (capacity) goes less than required level $\mathrm{x} 0$.

$\mathrm{c}_{\mathrm{i}} \quad$ probability that the fault is in the ith press, $\mathrm{i}=1,2,3,4$

$\mathrm{d}_{\mathrm{i}} \quad$ probability that the fault is in the ith glaze line, $\mathrm{i}=1,2,3,4$

$\mathrm{e}_{\mathrm{i}} \quad$ probability that the fault is in the ith kiln, $\mathrm{i}=1,2,3,4$

$\mathrm{I}_{3}(\mathrm{t}) / \mathrm{i}_{3}(\mathrm{t}) \quad$ c.d.f/p.d.f of inspection time for subsystem C

$\mathrm{I}_{4}(\mathrm{t}) / \mathrm{i}_{4}(\mathrm{t}) \quad$ c.d.f/p.d.f of inspection time for subsystem D

$\mathrm{I}_{5}(\mathrm{t}) / \mathrm{i}_{5}(\mathrm{t}) \quad$ c.d.f/p.d.f of inspection time for subsystem $\mathrm{E}$

$\mathrm{G}_{\mathrm{a} 1}(\mathrm{t}) / \mathrm{g}_{\mathrm{a} 1}(\mathrm{t})$ c.d.f/p.d.f of repair time for subsystem A when repair is done before the dust storage

$G_{a 2}(t) g_{a 2}(t) \quad$ c.d.f/p.d.f of repair time for subsystem A when repair is done after the dust storage

$\mathrm{G}_{\mathrm{b} 1}(\mathrm{t}) / \mathrm{g}_{\mathrm{b} 1}(\mathrm{t})$ c.d.f/p.d.f of repair time for subsystem B when repair is done before the dust storage

$\mathrm{G}_{\mathrm{b} 2}(\mathrm{t}) / \mathrm{g}_{\mathrm{b} 2}(\mathrm{t}) \quad$ c.d.f/p.d.f of repair time for subsystem B when repair is done after the dust storage

$\mathrm{G}_{\mathrm{c}}(\mathrm{t}) / \mathrm{g}_{\mathrm{c}}(\mathrm{t}) \quad$ c.d.f/p.d.f of repair time for subsystem C

$G_{d}(t) / g_{d}(t) \quad$ c.d.f/p.d.f. of repair time for subsystem $D$

$\mathrm{Ge}(\mathrm{t}) / \mathrm{ge}(\mathrm{t}) \quad$ c.d.f/p.d.f. of repair time for subsystem $\mathrm{E}$

\section{Transition Probabilities}

Figure 1 shows possible states of transitions of the system. The points of entry into states $0,1,2,3,4,5,6,7$,
$8,9,10,11,12,13,14,15,16,17,18$ and 19 are regeneration points and hence these are regenerative states. The states 2 and 4 are failed states and 5, 6, 7, 8, 9, 10, 11, $12,13,14,15,16,17,18$ and 19 are degraded states. The transition probabilities are stated as

$$
\begin{aligned}
& q_{01}(t)=p_{1} \lambda_{1} e^{-\left(\lambda_{1}+\lambda_{2}+\lambda_{3}+\lambda_{4}+\lambda_{5}\right)}, \\
& q_{02}(t)=q_{1} \lambda_{1} e^{-\left(\lambda_{1}+\lambda_{2}+\lambda_{3}+\lambda_{4}+\lambda_{5}\right)}, \\
& q_{03}(t)=p_{1} \lambda_{2} e^{-\left(\lambda_{1}+\lambda_{2}+\lambda_{3}+\lambda_{4}+\lambda_{5}\right)}, \\
& q_{04}(t)=q_{1} \lambda_{2} e^{-\left(\lambda_{1}+\lambda_{2}+\lambda_{3}+\lambda_{4}+\lambda_{5}\right)}, \\
& q_{05}(t)=\lambda_{3} e^{-\left(\lambda_{1}+\lambda_{2}+\lambda_{3}+\lambda_{4}+\lambda_{5}\right)}, \\
& q_{06}(t)=\lambda_{4} e^{-\left(\lambda_{1}+\lambda_{2}+\lambda_{3}+\lambda_{4}+\lambda_{5}\right)}, \\
& q_{07}(t)=\lambda_{5} e^{-\left(\lambda_{1}+\lambda_{2}+\lambda_{3}+\lambda_{4}+\lambda_{5}\right)}, \\
& q_{12}(t)=q_{2} g_{a_{1}}(t), q_{34}(t)=q_{2} g_{b_{1}}(t), q_{10}(t)=p_{2} g_{a_{1}}(t) \\
& q_{30}(t)=p_{2} g_{b_{1}}(t), q_{20}(t)=g_{a_{2}}(t) \\
& q_{40}(t)=g_{b_{2}}(t), q_{58}(t)=q_{3} c_{1} i_{3}(t) \\
& q_{59}(t)=q_{3} c_{2} i_{3}(t), q_{5,10}(t)=q_{3} c_{3} i_{3}(t) \\
& q_{5,11}(t)=q_{3} c_{4} i_{3}(t), q_{6,12}(t)=q_{4} d_{1} i_{4}(t) \\
& q_{6,13}(t)=q_{4} d_{2} i_{4}(t), q_{6,14}(t)=q_{4} d_{3} i_{4}(t) \\
& q_{6,15}(t)=q_{4} d_{4} i_{4}(t), q_{7,16}(t)=e_{1} i_{5}(t) \\
& q_{7,17}(t)=e_{2} i_{5}(t), q_{7,18}(t)=e_{3} i_{5}(t) \\
& q_{7,19}(t)=e_{4} i_{5}(t), q_{80}(t)=g_{c}(t) \\
& q_{90}(t)=g_{c}(t), q_{10,0}(t)=g_{c}(t) \\
& q_{11,0}(t)=g_{c}(t), q_{12,0}(t)=g_{d}(t) \\
& q_{13,0}(t)=g_{d}(t), q_{14,0}(t)=g_{d}(t), q_{15,0}(t)=g_{d}(t), \\
& q_{16,0}(t)=g_{e}(t), q_{17,0}(t)=g_{e}(t), q_{18,0}(t)=g_{e}(t), \\
& q_{19,0}(t)=g_{e}(t), q_{56}(t)=p_{3} i_{3}(t), q_{67}(t)=p_{4} i_{4}(t) .
\end{aligned}
$$

The non-zero elements $p_{i j}$ are acquired as given $p_{i j}=\lim _{s \rightarrow 0} q_{i j}^{*}(s)=\lim _{s \rightarrow 0} Q_{i j}^{* *}(s)$.

$$
\begin{aligned}
& p_{01}=\frac{p_{1} \lambda_{1}}{\lambda_{1}+\lambda_{2}+\lambda_{3}+\lambda_{4}+\lambda_{5}}, p_{02}=\frac{q_{1} \lambda_{1}}{\lambda_{1}+\lambda_{2}+\lambda_{3}+\lambda_{4}+\lambda_{5}} \\
& p_{03}=\frac{p_{1} \lambda_{2}}{\lambda_{1}+\lambda_{2}+\lambda_{3}+\lambda_{4}+\lambda_{5}}, p_{04}=\frac{q_{1} \lambda_{2}}{\lambda_{1}+\lambda_{2}+\lambda_{3}+\lambda_{4}+\lambda_{5}} \\
& p_{05}=\frac{\lambda_{3}}{\lambda_{1}+\lambda_{2}+\lambda_{3}+\lambda_{4}+\lambda_{5}}, p_{06}=\frac{\lambda_{4}}{\lambda_{1}+\lambda_{2}+\lambda_{3}+\lambda_{4}+\lambda_{5}} \\
& p_{07}=\frac{\lambda_{5}}{\lambda_{1}+\lambda_{2}+\lambda_{3}+\lambda_{4}+\lambda_{5}}, p_{10}=p_{2} g_{a_{1}}^{*}(0) \\
& p_{30}=p_{2} g_{b_{1}}^{*}(0), p_{12}=q_{2} g_{a_{1}}^{*}(0), \\
& p_{34}=q_{2} g_{b_{1}}^{*}(0), p_{58}=q_{3} c_{1} i_{3}^{*}(0) .
\end{aligned}
$$

$$
\begin{aligned}
& p_{59}=q_{3} c_{2} i_{3}^{*}(0), p_{5,10}=q_{3} c_{3} i_{3}^{*}(0), p_{5,11}=q_{3} c_{4} i_{3}^{*}(0), \\
& p_{6,12}=q_{4} d_{1} i_{4}^{*}(0), p_{6,13}=q_{4} d_{2} i_{4}^{*}(0), p_{6,14}=q_{4} d_{3} i_{4}^{*}(0) \\
& p_{6,15}=q_{4} d_{4} i_{4}^{*}(0), p_{7,16}=e_{1} i_{5}^{*}(0), p_{7,17}=e_{2} i_{5}^{*}(0),
\end{aligned}
$$




$$
\begin{aligned}
& p_{7,18}=e_{3} i_{5}^{*}(0), p_{7,19}=e_{4} i_{5}^{*}(0), \\
& p_{56}=p_{3} i_{3}^{*}(0) p_{67}=p_{4} i_{4}^{*}(0), \\
& p_{80}=p_{90}=p_{10,0}=p_{11,0}=g_{c}^{*}(0) \\
& p_{12,0}=p_{13,0}=p_{14,0}=p_{15,0}=g_{d}^{*}(0), \\
& p_{16,0}=p_{17,0}=p_{18,0}=p_{19,0}=g_{e}^{*}(0) .
\end{aligned}
$$

Clearly, it can be justified that

$$
\begin{aligned}
& p_{01}+p_{02}+p_{03}+p_{04}+p_{05}+p_{06}+p_{07}=1, \\
& p_{10}+p_{12}=1, p_{30}+p_{34}=1, \\
& p_{56}+p_{58}+p_{59}+p_{5,10}+p_{5,11}=1 \\
& p_{67}+p_{6,12}+p_{6,13}+p_{6,14}+p_{6,15}=1, \\
& p_{7,16}+p_{7,17}+p_{7,18}+p_{7,19}=1
\end{aligned}
$$
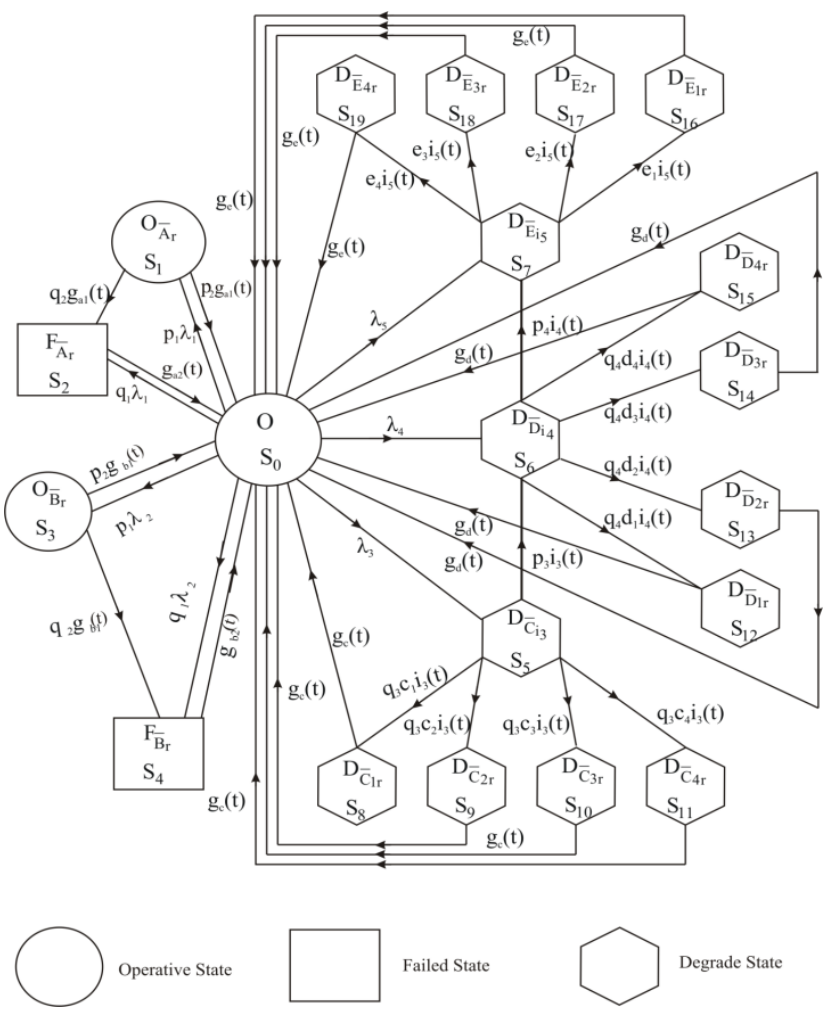

Figure 1. State Transition Diagram

\section{Mean Sojourn Time}

Mean sojourn time $\left(\mu_{\mathrm{i}}\right)$ is the mean first passage time taken by the ith state before transiting to any other state.

$$
\mu_{\mathrm{i}}=\int_{0}^{\infty} \operatorname{Pr}\left(T_{i}>t\right) d t
$$

where $T_{i}$ is the p.d.f. of device life span.

$$
\begin{aligned}
& \mu_{0}=\frac{1}{\lambda_{1}+\lambda_{2}+\lambda_{3}+\lambda_{4}+\lambda_{5}}, \\
& \mu_{1}=-g_{a_{1}}^{*^{\prime}}(0), \mu_{2}=-g_{a_{2}}^{*^{\prime}}(0), \mu_{3}=-g_{b_{1}}^{*^{\prime}}(0), \\
& \mu_{4}=-g_{b_{2}}^{*^{\prime}}(0), \mu_{5}=-i_{3}^{*^{\prime}}(0), \mu_{6}=-i_{4}^{*^{\prime}}(0),
\end{aligned}
$$

$$
\begin{aligned}
& \mu_{7}=-i_{5}^{*^{\prime}}(0), \mu_{8}=\mu_{9}=\mu_{10}=\mu_{11}=-g_{c}^{*^{\prime}}(0) \\
& \mu_{12}=\mu_{13}=\mu_{14}=\mu_{15}=-g_{d}^{*^{\prime}}(0), \\
& \mu_{16}=\mu_{17}=\mu_{18}=\mu_{19}=-g_{e}^{*^{\prime}}(0) .
\end{aligned}
$$

The unconditional mean time obtained by the system to transit for any state $\mathrm{j}$ when it is calculated from the point of entrance into the state $i$, is mathematically expressed as:

$$
m_{i j}=\int_{0}^{\infty} t q_{i j}(t) d t=-q_{i j}^{*^{\prime}}(0)
$$

thus

$$
\begin{aligned}
& m_{01}=\frac{p_{1} \lambda_{1}}{\left(\lambda_{1}+\lambda_{2}+\lambda_{3}+\lambda_{4}+\lambda_{5}\right)^{2}}, \\
& m_{02}=\frac{\mathrm{q}_{1} \lambda_{1}}{\left(\lambda_{1}+\lambda_{2}+\lambda_{3}+\lambda_{4}+\lambda_{5}\right)^{2}}, \\
& m_{03}=\frac{p_{1} \lambda_{2}}{\left(\lambda_{1}+\lambda_{2}+\lambda_{3}+\lambda_{4}+\lambda_{5}\right)^{2}} \text {, } \\
& m_{04}=\frac{\mathrm{q}_{1} \lambda_{2}}{\left(\lambda_{1}+\lambda_{2}+\lambda_{3}+\lambda_{4}+\lambda_{5}\right)^{2}}, \\
& m_{05}=\frac{\lambda_{3}}{\left(\lambda_{1}+\lambda_{2}+\lambda_{3}+\lambda_{4}+\lambda_{5}\right)^{2}}, \\
& m_{06}=\frac{\lambda_{4}}{\left(\lambda_{1}+\lambda_{2}+\lambda_{3}+\lambda_{4}+\lambda_{5}\right)^{2}}, \\
& m_{07}=\frac{\lambda_{5}}{\left(\lambda_{1}+\lambda_{2}+\lambda_{3}+\lambda_{4}+\lambda_{5}\right)^{2}}, \\
& m_{10}=-p_{2} g_{a_{1}}^{*^{\prime}}(0), m_{12}=-q_{2} g_{a_{1}}^{*^{\prime}}(0) \text {, } \\
& m_{30}=-p_{2} g_{b_{1}}^{*^{\prime}}(0), m_{34}=-q_{2} g_{b_{1}}^{*^{\prime}}(0) \text {, } \\
& m_{40}=-g_{b_{2}}^{*^{\prime}}(0), m_{56}=-p_{3} c_{1} i_{3}^{*^{\prime}}(0) \text {, } \\
& m_{58}=-q_{3} C_{1} i_{3}^{*^{\prime}}(0), m_{59}=-q_{3} C_{2} i_{3}^{*^{\prime}}(0) \text {, } \\
& m_{5,10}=-q_{3} c_{3} i_{3}^{*^{\prime}}(0), m_{5,11}=-q_{3} c_{4} i_{3}^{*^{\prime}}(0), \\
& m_{67}=-p_{4} i_{4}^{*^{\prime}}(0), m_{6,12}=-q_{4} c_{1} i_{4}^{*^{\prime}}(0), \\
& m_{6,13}=-q_{4} c_{2} i_{4}^{*^{\prime}}(0), m_{6,14}=-q_{4} c_{3} i_{4}^{*^{\prime}}(0) \text {, } \\
& m_{6,15}=-q_{4} c_{4} i_{4}^{*^{\prime}}(0), m_{7,16}=-c_{1} i_{5}^{*^{\prime}}(0), \\
& m_{7,17}=-c_{2} i_{5}^{*^{\prime}}(0), m_{7,18}=-c_{3} i_{5}^{*^{\prime}}(0) \text {, } \\
& m_{7,19}=-c_{4} i_{5}^{*^{\prime}}(0), \\
& m_{80}=m_{90}=m_{10,0}=m_{11,0}=-g_{c}^{*}(0), \\
& m_{12,0}=m_{13,0}=m_{14,0}=m_{15,0}=-g_{d}^{*^{\prime}}(0) \text {, } \\
& m_{16,0}=m_{17,0}=m_{18,0}=m_{19,0}=-g_{e}^{*^{\prime}}(0) \text {. }
\end{aligned}
$$


It is certified that

$$
\begin{aligned}
& m_{01}+m_{02}+m_{03}+m_{04}+m_{05}+m_{06}+m_{07}=\mu_{0} \\
& m_{10}+m_{12}=\mu_{1}, m_{20}=\mu_{2}, m_{30}+m_{34}=\mu_{3}, \\
& m_{40}=\mu_{4}, m_{56}+m_{58}+m_{59}+m_{5,10}+m_{5,11}=\mu_{5} \\
& m_{67}+m_{6,12}+m_{6,13}+m_{6,14}+m_{6,15}=\mu_{6}, \\
& m_{7,16}+m_{7,17}+m_{7,18}+m_{7,19}=\mu_{7}, \\
& m_{80}=\mu_{9}, m_{90}=\mu_{9}, m_{10,0}=\mu_{10}, m_{11,0}=\mu_{11} .
\end{aligned}
$$

\section{Mean Time to System Failure}

MTSF is determined regarding the failed states as absorbing states of the system. Using probabilistic arguments, following are the recurrence relations for $\phi_{\mathrm{i}}(\mathrm{t})$, c.d.f of the first passage time from regenerative state 'i' to failed state:

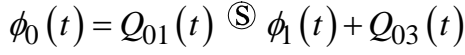

$$
\begin{aligned}
& \text { (S) } \phi_{3}(t)+Q_{02}(t)+Q_{04}(t)+Q_{05}(t)
\end{aligned}
$$

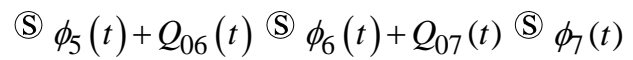

$$
\begin{aligned}
& \phi_{1}(t)=Q_{10}(t) \stackrel{(S)}{S} \phi_{0}(t)+Q_{12}(t) \\
& \phi_{3}(t)=Q_{30}(t) \stackrel{(S)}{ } \phi_{0}(t)+Q_{34}(t)
\end{aligned}
$$

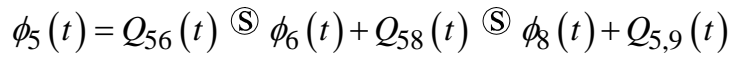

$$
\begin{aligned}
& \text { (S) } \phi_{9}(t)+Q_{5,10}(t) \stackrel{(\mathbf{S}}{ } \phi_{10}(t)+Q_{5,11}(t) \stackrel{(\mathbf{S}}{ } \phi_{11}(t) \\
& \phi_{6}(t)=Q_{6,7}(t) \stackrel{S}{S} \phi_{7}(t)+Q_{6,12}(t)
\end{aligned}
$$

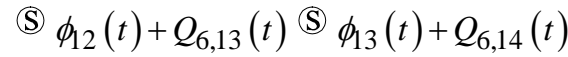

$$
\begin{aligned}
& \text { (S) } \phi_{14}(t)+Q_{6,15}(t) \text { (S) } \phi_{15}(t) \\
& \phi_{7}(t)=Q_{7,16}(t) \stackrel{S}{S} \phi_{16}(t)+Q_{7,17}(t) \\
& \text { (S) } \phi_{17}(t)+Q_{7,18}(t) \text { (S) } \phi_{18}(t)+Q_{7,19}(t) \text { (S) } \phi_{19}(t) \\
& \phi_{8}(t)=Q_{80}(t) \stackrel{(S)}{S} \phi_{0}(t) \\
& \phi_{9}(t)=Q_{90}(t) \stackrel{(\mathbf{S}}{S} \phi_{0}(t) \\
& \phi_{10}(t)=Q_{10,0}(t) \stackrel{(S)}{S} \phi_{0}(t) \\
& \phi_{11,0}(t)=Q_{11,0}(t) \stackrel{(S)}{S} \phi_{0}(t) \\
& \phi_{12}(t)=Q_{12,0}(t) \stackrel{(S)}{ } \phi_{0}(t) \\
& \phi_{13}(t)=Q_{13,0}(t) \stackrel{(S)}{ } \phi_{0}(t) \\
& \phi_{14}(t)=Q_{14,0}(t) \stackrel{(S)}{S} \phi_{0}(t) \\
& \phi_{15}(t)=Q_{15,0}(t) \stackrel{(S)}{ } \phi_{0}(t) \\
& \phi_{16}(t)=Q_{16,0}(t) \stackrel{(S)}{S} \phi_{0}(t) \\
& \phi_{17}(t)=Q_{17,0}(t) \stackrel{(S)}{S} \phi_{0}(t) \\
& \phi_{18}(t)=Q_{18,0}(t) \stackrel{(S)}{ } \phi_{0}(t) \\
& \phi_{19}(t)=Q_{19,0}(t) \stackrel{(S)}{ } \phi_{0}(t) \text {. }
\end{aligned}
$$

Taking L.S.T. of these equations and after solving for $\phi_{0}^{* *}(s)$, we obtain

$$
\phi_{0}^{* *}(s)=\frac{N(s)}{D(s)} .
$$

The mean time to system failure when the system starts from the state 0 , is

$$
\mathrm{MTSF}=\lim _{s \rightarrow 0} \frac{1-\phi_{0}^{* *}(s)}{s}=\frac{N}{D}
$$

where

$$
\begin{aligned}
& N=\mu_{0}+p_{01} \mu_{1}+p_{03} \mu_{3} \\
& +p_{05}\left(\mu_{5}+p_{56} \mu_{6}+p_{56} p_{67} \mu_{7}+p_{58} \mu_{8}\right. \\
& +p_{58} \mu_{8}+p_{59} \mu_{9}+p_{5,10} \mu_{10}+p_{5,11} \mu_{11} \\
& +p_{56} p_{612} \mu_{12}+p_{56} p_{613} \mu_{13}+p_{56} p_{6714} \mu_{14} \\
& +p_{56} p_{615} \mu_{15}+p_{56} p_{67} p_{716} \mu_{16} \\
& +p_{56} p_{67} p_{717} \mu_{17}+p_{56} p_{67} p_{718} \mu_{18} \\
& \left.+p_{56} p_{67} p_{719} \mu_{19}\right) \\
& +p_{06}\left(\mu_{6}+p_{67} \mu_{7}+p_{6,12} \mu_{12}+p_{6,13} \mu_{13}\right. \\
& +p_{6,14} \mu_{14}+p_{6,15} \mu_{15}+p_{67} p_{7,16} \mu_{16} \\
& \left.+p_{67} p_{7,17} \mu_{17}+p_{67} p_{7,18} \mu_{18}+p_{67} p_{7,19} \mu_{19}\right) \\
& +p_{07}\left(\mu_{7}+p_{7,16} \mu_{16}+p_{7,17} \mu_{17}+p_{7,18} \mu_{18}+p_{7,19} \mu_{19}\right) . \\
& \text { and } \mathrm{D}=1-\mathrm{p}_{01} \mathrm{p}_{10}-\mathrm{p}_{03} \mathrm{p}_{30}-\mathrm{p}_{05}-\mathrm{p}_{06}-\mathrm{p}_{07 .}
\end{aligned}
$$

\section{Availability of the System with Full Capacity}

By the assertions of the theory of regenerative processes, the availability of the system with full capacity $\left(A F_{i}(t)\right)$, the probability that the system is working at instant ' $t$ ' with full capacity given that it entered regenerative state ' $\mathrm{i}$ ' at $\mathrm{t}=0$, is seen to satisfy the following recurrence relations.

$$
\begin{aligned}
& A F_{0}(t)=M_{0}(t)+q_{01}(t) \Subset A F_{1}(t)+q_{02}(t) \Subset A F_{2}(t) \\
& +q_{03}(t) \Subset A F_{3}(t)+q_{04}(t) \Subset A F_{4}(t) \\
& +q_{05}(t) \Subset A F_{5}(t)+q_{06}(t) \Subset A F_{6}(t) \\
& +q_{07}(t) \Subset A F_{7}(t) \\
& A F_{1}(t)=M_{1}(t)+q_{10}(t) \subseteq A F_{0}(t)+q_{12}(t) \subseteq A F_{2}(t) \\
& A F_{2}(t)=q_{20}(t) \oplus A F_{0}(t) \\
& A F_{3}(t)=M_{3}(t)+q_{30}(t) \subseteq A F_{0}(t)+q_{34}(t) \subseteq A F_{4}(t) \\
& A F_{4}(t)=q_{40}(t) \subseteq A F_{0}(t) \\
& A F_{5}(t)=q_{56} \Subset A F_{6}+q_{58}(t) \Subset A F_{8}(t)+q_{59}(t) \Subset A F_{9}(t) \\
& +q_{5,10}(t) \Subset A F_{10}(t)+q_{5,11}(t) \Subset A F_{11}(t) \\
& A F_{6}(t)=q_{67}(t) \Subset A F_{7}(t)+q_{6,12}(t) \Subset A F_{12}(t) \\
& +q_{6,13}(t) \oplus A F_{13}(t)+q_{6,14}(t) \oplus A F_{14}(t) \\
& +q_{6,15}(t) \subset A F_{15}(t) \\
& A F_{7}(t)=q_{7,16}(t) \subseteq A F_{16}(t)+q_{7,17}(t) \subseteq A F_{17}(t) \\
& +q_{7,18}(t) \Subset A F_{18}(t)+q_{7,19}(t) \Subset A F_{19}(t)
\end{aligned}
$$




$$
\begin{aligned}
A F_{8}(t) & =q_{80}(t) \odot A F_{0}(t) \\
A F_{9}(t) & =q_{90}(t) \odot A F_{0}(t) \\
A F_{10}(t) & =q_{10,0}(t) \odot A F_{0}(t) \\
A F_{11}(t) & =q_{11,0}(t) \odot A F_{0}(t) \\
A F_{12}(t) & =q_{12,0}(t) \odot A F_{0}(t) \\
A F_{13}(t) & =q_{13,0}(t) \odot A F_{0}(t) \\
A F_{14}(t) & =q_{14,0}(t) \odot A F_{0}(t) \\
A F_{15}(t) & =q_{15,0}(t) \odot A F_{0}(t) \\
A F_{16}(t) & =q_{16,0}(t) \odot A F_{0}(t) \\
A F_{17}(t) & =q_{17,0}(t) \odot A F_{0}(t) \\
A F_{18}(t) & =q_{18,0}(t) \odot A F_{0}(t) \\
A F_{19}(t) & =q_{19,0}(t) \odot A F_{0}(t)
\end{aligned}
$$

where

$$
\begin{gathered}
M_{0}(t)=e^{-\left(\lambda_{1}+\lambda_{2}+\lambda_{3}+\lambda_{4}+\lambda_{5}\right) t} \\
M_{1}(t)=\overline{G_{a_{1}}}(t), M_{3}(t)=\overline{G_{b_{1}}}(t) .
\end{gathered}
$$

Taking Laplace transform of the above equations and solving for $A F_{0}^{*}(s)$, we have

$$
A F_{0}^{*}(s)=\frac{N_{1}(s)}{D_{1}(s)} .
$$

The steady state availability of the system with full capacity is given by

$$
A F_{0}=\lim _{s \rightarrow 0}\left(s A F_{0}^{*}(s)\right)=\frac{N_{1}}{D_{1}}
$$

where

$$
\begin{gathered}
N_{1}=\mu_{0}+p_{01} \mu_{1}+p_{03} \mu_{3} \\
\begin{array}{l}
D_{1}=\mu_{0}+p_{01} \mu_{1}+\left(p_{01} p_{12}+p_{02}\right) \mu_{2} \\
+p_{03} \mu_{3}+\left(p_{03} p_{34}+p_{04}\right) \mu_{4}
\end{array} \\
\left.+\begin{array}{l}
\mu_{5}+p_{56} \mu_{6}+p_{56} p_{67} \mu_{7}+p_{58} \mu_{8} \\
+p_{58} \mu_{8}+p_{59} \mu_{9}+p_{5,10} \mu_{10}+p_{5,11} \mu_{11} \\
+p_{56} p_{612} \mu_{12}+p_{56} p_{613} \mu_{13} \\
+p_{56} p_{6714} \mu_{14}+p_{56} p_{615} \mu_{15} \\
+p_{56} p_{67} p_{716} \mu_{16}+p_{56} p_{67} p_{717} \mu_{17} \\
+p_{56} p_{67} p_{718} \mu_{18}+p_{56} p_{67} p_{719} \mu_{19}
\end{array}\right) \\
+p_{06} \mu_{6}+p_{67} \mu_{7}+p_{6,12} \mu_{12}+p_{6,13} \mu_{13} \\
+p_{6,14} \mu_{14}+p_{6,15} \mu_{15}+p_{67} p_{7,16} \mu_{16} \\
\left.+p_{67} p_{7,17} \mu_{17}+p_{67} p_{7,18} \mu_{18}+p_{67} p_{7,19} \mu_{19}\right) \\
+p_{07}\left(\begin{array}{l}
\mu_{7}+p_{7,16} \mu_{16}+p_{7,17} \mu_{17} \\
+p_{7,18} \mu_{18}+p_{7,19} \mu_{19}
\end{array}\right) .
\end{gathered}
$$

\section{Other Measures of System Effectiveness}

Availability of the system with reduced capacity $=\frac{N_{2}}{D_{1}}$ Expected number of repairs/replacements in ball mill $=\frac{N_{3}}{D_{1}}$

Expected number of repairs/replacements in spray dryer $=\frac{N_{4}}{D_{1}}$

Expected number of inspections in hydraulic press $=\frac{N_{5}}{D_{1}}$

Expected number of repairs/replacements in hydraulic press $=\frac{N_{6}}{D_{1}}$

Expected number of inspections in glaze line $=\frac{N_{7}}{D_{1}}$

Expected number of repairs/replacements in glaze line $=\frac{N_{8}}{D_{1}}$

Expected number of inspections in kiln $=\frac{N_{9}}{D_{1}}$

Expected number of repairs/replacements in kiln $=\frac{N_{10}}{D_{1}}$

Expected number of visit by the repairman $=\frac{N_{11}}{D_{1}}$,

$$
N_{2}=p_{05}\left(\begin{array}{l}
\mu_{5}+p_{56} \mu_{6}+p_{56} p_{67} \mu_{7}+p_{58} \mu_{8} \\
+p_{58} \mu_{8}+p_{59} \mu_{9}+p_{5,10} \mu_{10} \\
+p_{5,11} \mu_{11}+p_{56} p_{612} \mu_{12}+p_{56} p_{613} \mu_{13} \\
+p_{56} p_{6714} \mu_{14}+p_{56} p_{615} \mu_{15} \\
+p_{56} p_{67} p_{716} \mu_{16}+p_{56} p_{67} p_{717} \mu_{17} \\
+p_{56} p_{67} p_{718} \mu_{18}+p_{56} p_{67} p_{719} \mu_{19}
\end{array}\right)
$$

$+p_{06}\left(\begin{array}{l}\mu_{6}+p_{67} \mu_{7}+p_{6,12} \mu_{12}+p_{6,13} \mu_{13}+p_{6,14} \mu_{14} \\ +p_{6,15} \mu_{15}+p_{67} p_{7,16} \mu_{16}+p_{67} p_{7,17} \mu_{17} \\ +p_{67} p_{7,18} \mu_{18}+p_{67} p_{7,19} \mu_{19}\end{array}\right)$

$+p_{07}\left(\mu_{7}+p_{7,16} \mu_{16}+p_{7,17} \mu_{17}+p_{7,18} \mu_{18}+p_{7,19} \mu_{19}\right)$

$N_{3}=p_{01}+p_{02}+p_{01} p_{12}$

$N_{4}=p_{03}+p_{04}+p_{03} p_{34}$

$N_{5}=p_{05}+p_{05} p_{58}+p_{05} p_{59}+p_{05} p_{5,10}+p_{05} p_{5,11}$

$N_{6}=p_{05}+p_{05} p_{58}+p_{05} p_{59}+p_{05} p_{5,10}+p_{05} p_{5,11}$

$N_{7}=p_{06}+p_{06} p_{6,12}+p_{06} p_{6,13}+p_{06} p_{6,14}+p_{06} p_{6,15}$

$N_{8}=p_{06}+p_{06} p_{6,12}+p_{06} p_{6,13}+p_{06} p_{6,14}+p_{06} p_{6,15}$

$N_{9}=p_{07}+p_{07} p_{7,16}+p_{07} p_{7,17}+p_{07} p_{7,18}+p_{07} p_{7,19}$

$N_{10}=p_{07}+p_{07} p_{7,16}+p_{07} p_{7,17}+p_{07} p_{7,18}+p_{07} p_{7,19}$

$N_{11}=p_{01}+p_{02}+p_{03}+p_{04}+p_{05}+p_{06}+p_{07}$

and $\mathrm{D}_{1}$ is already defined. 


\section{Profit Analysis of the System}

Expected Profit incurred to the System is given as

$$
\begin{aligned}
P= & K_{0}\left(F A V_{0}+R A V_{0}\right)-K_{1} R A_{0}-K_{2} R B_{0} \\
& -K_{3} I C_{0}-K_{4} R C_{0}-K_{5} I D_{0}-K_{6} R D_{0} \\
& -K_{7} I E_{0}-K_{8} R E_{0}-K_{9} V_{0}
\end{aligned}
$$

$\mathrm{K}_{0}=$ revenue per unit up time of the system

$\mathrm{K}_{1}=$ cost per repair/replacement of parts in the subsystem ball mill

$\mathrm{K}_{2}$ = cost per repair/replacement of parts in the subsystem spray dryer

$\mathrm{K}_{3}=$ cost per inspection of parts in the subsystem hydraulic press

$\mathrm{K}_{4}=$ cost per repair/replacement of parts in the subsystem hydraulic press

$\mathrm{K}_{5}=$ cost per inspection of parts in the subsystem glaze line

$\mathrm{K}_{6}=$ cost per repair/replacement of parts in the subsystem glaze line

$\mathrm{K}_{7}=$ cost per inspection of parts in the subsystem kiln

$\mathrm{K}_{8}=$ cost per repair/replacement of parts in the subsystem kiln

$\mathrm{K}_{9}=$ cost per visit of the repairman

\section{Numerical Computation and Graphical Interpretations}

The following particular cases are considered for the analysis purpose

$$
\begin{aligned}
& \mathrm{g}_{\mathrm{a}_{1}}(\mathrm{t})=\beta_{\mathrm{a}_{1}} \mathrm{e}^{-\beta_{\mathrm{a}_{1}} t}, \mathrm{~g}_{a_{2}}(\mathrm{t})=\beta_{\mathrm{a}_{2}} \mathrm{e}^{-\beta_{\mathrm{a}_{2}} t}, \mathrm{~g}_{b_{1}}(\mathrm{t})=\beta_{b_{1}} \mathrm{e}^{-\beta_{b_{1}} t} \\
& \mathrm{~g}_{b_{2}}(\mathrm{t})=\beta_{b_{2}} \mathrm{e}^{-\beta_{b_{2}} t}, \mathrm{~g}_{\mathrm{c}}(\mathrm{t})=\beta_{\mathrm{c}} \mathrm{e}^{-\beta_{\mathrm{c}} t}, \mathrm{~g}_{\mathrm{d}}(\mathrm{t})=\beta_{\mathrm{d}} \mathrm{e}^{-\beta_{\mathrm{d}} t} \\
& \mathrm{~g}_{\mathrm{e}}(\mathrm{t})=\beta_{\mathrm{e}} \mathrm{e}^{-\beta_{\mathrm{e}} t}, i_{3}(t)=\alpha_{3} e^{-\alpha_{3} t}, i_{4}(t)=\alpha_{4} e^{-\alpha_{4} t} \\
& i_{5}(t)=\alpha_{5} e^{-\alpha_{5} t}
\end{aligned}
$$

Several graphs have been depicted for mean time to system failure and profit of the system for distinct values of failure rates $\left(\lambda_{1}, \lambda_{2}, \lambda_{3}, \lambda_{4}, \lambda_{5}\right)$, repair and inspection rates $\left(\beta_{\mathrm{a}_{1}}, \beta_{\mathrm{a}_{2}}, \beta_{b_{1}}, \beta_{b_{2}}, \beta_{\mathrm{c}}, \beta_{\mathrm{d}}, \beta_{\mathrm{e}}, \alpha_{3}, \alpha_{4}, \alpha_{5}\right)$, probabilities $\left(\mathrm{q}_{1}, \mathrm{q}_{2}, \mathrm{p}_{1}, \mathrm{p}_{2}\right)$ and costs $\left(\mathrm{K}_{0}, \mathrm{~K}_{1}, \mathrm{~K}_{2}, \mathrm{~K}_{3}, \mathrm{~K}_{4}\right.$, $\mathrm{K}_{5}, \mathrm{~K}_{6}, \mathrm{~K}_{7}, \mathrm{~K}_{8}, \mathrm{~K}_{9}$ ).

Figure 2 gives the graphs between mean time to system failure and the rate of occurrence of faults $\left(\lambda_{2}\right)$ in subsystem B for distinct values of repair rate $\left(\beta_{b 1}\right)$. The graph depicts that the MTSF declined with increment in the values of rates of occurrence of faults in subsystem B and having larger values for high values of repair rate $\left(\beta_{\mathrm{b} 1}\right)$. The values of various other parameters are $\lambda_{2}=0.0002 / \mathrm{hr}$, $\lambda_{3}=0.00025 / \mathrm{hr}, \lambda_{4}=0.00019 / \mathrm{hr}, \lambda_{5}=0.0039 / \mathrm{hr}, \beta_{\mathrm{a} 1}=3 / \mathrm{hr}$, $\beta_{\mathrm{a} 2}=3.5 / \mathrm{hr}, \beta_{\mathrm{b} 2}=3.5 / \mathrm{hr}, \beta_{\mathrm{b} 2}=4 / \mathrm{hr}, \beta_{\mathrm{c}}=2 / \mathrm{hr}, \beta_{\mathrm{d}}=2.3 / \mathrm{hr}, \beta_{\mathrm{e}}$ $=2 / \mathrm{hr}, \alpha_{3}=1 / \mathrm{hr}, \alpha_{4}=1.3 / \mathrm{hr}, \alpha_{5}=1.8 / \mathrm{hr}, \mathrm{p}_{2}=0.4$.

Figure 3 gives the graphs between availability with reduced capacity and the rate of occurrence of faults $\left(\lambda_{1}\right)$ in subsystem $A$ for different values of repair rate $\left(\beta_{\mathrm{a} 1}\right)$. The graph reveals that the availability decreases with increase in the values of rates of occurrence of faults in subsystem $A$ and having higher values for high values of repair rate $\left(\beta_{\mathrm{a} 1}\right)$. The values of various other parameters are $\lambda_{2}=0.0002 / \mathrm{hr}, \lambda_{3}=0.00025 / \mathrm{hr}, \lambda_{4}=0.00019 / \mathrm{hr}$, $\lambda_{5}=0.0039 / \mathrm{hr}, \beta_{\mathrm{a} 1}=3 / \mathrm{hr}, \beta_{\mathrm{a} 2}=3.5 / \mathrm{hr}, \beta_{\mathrm{b} 1}=3.5 / \mathrm{hr}$, $\beta_{\mathrm{b} 2}=4 / \mathrm{hr}, \beta_{\mathrm{c}}=2 / \mathrm{hr}, \beta_{\mathrm{d}}=2.3 / \mathrm{hr}, \quad \beta_{\mathrm{e}}=2 / \mathrm{hr}, \alpha_{3}=1 / \mathrm{hr}$, $\alpha_{4}=1.3 / \mathrm{hr}, \alpha_{5}=1.8 / \mathrm{hr}, \mathrm{p}_{2}=0.4$.

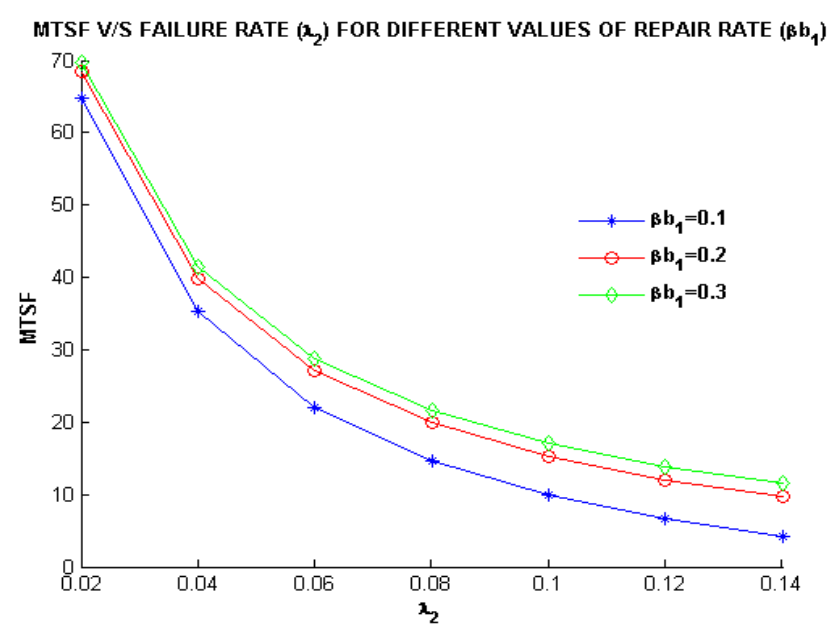

Figure 2.

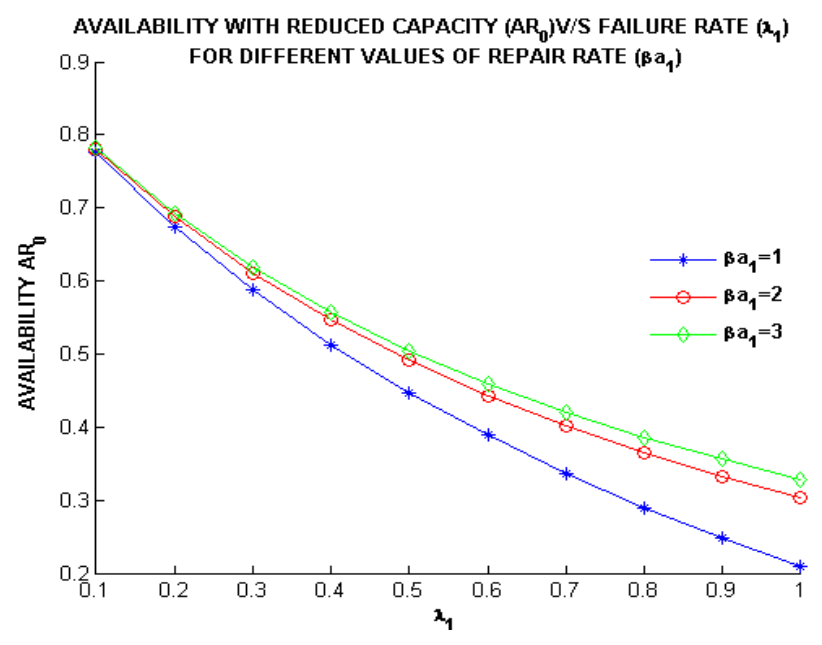

Figure 3.

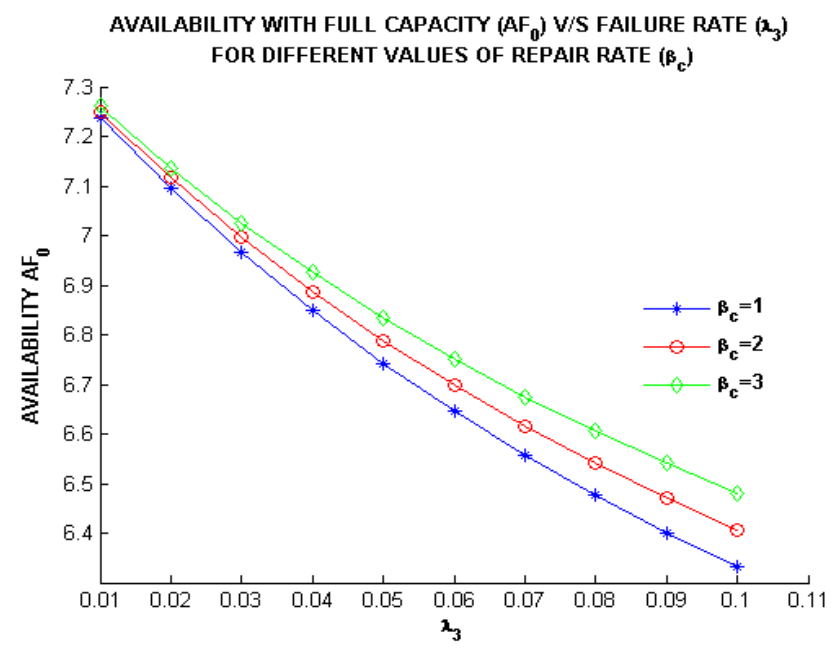

Figure 4. 
Figure 4 gives the graphs between availability with full capacity and the rate of occurrence of faults $\left(\lambda_{3}\right)$ in subsystem $C$ for distinct values of repair rate $\left(\beta_{c}\right)$. The graph reveals that the availability declines with increase in the values of rates of occurrence of faults in subsystem $C$ and having higher values for high values of repair rate $\left(\beta_{\mathrm{c}}\right)$. The values of various other parameters are $\lambda_{2}=0.0002 / \mathrm{hr}, \lambda_{3}=0.00025 / \mathrm{hr}, \lambda_{4}=0.00019 / \mathrm{hr}$, $\lambda_{5}=0.0039 / \mathrm{hr}, \beta_{\mathrm{a} 1}=3 / \mathrm{hr}, \beta_{\mathrm{a} 2}=3.5 / \mathrm{hr}, \beta_{\mathrm{b} 1}=3.5 / \mathrm{hr}$, $\beta_{\mathrm{b} 2}=4 / \mathrm{hr}, \beta_{\mathrm{c}}=2 / \mathrm{hr}, \beta_{\mathrm{d}}=2.3 / \mathrm{hr}, \beta_{\mathrm{e}}=2 / \mathrm{hr}, \alpha_{3}=1 / \mathrm{hr}$, $\alpha_{4}=1.3 / \mathrm{hr}, \alpha_{5}=1.8 / \mathrm{hr}, \mathrm{p}_{2}=0.4$.

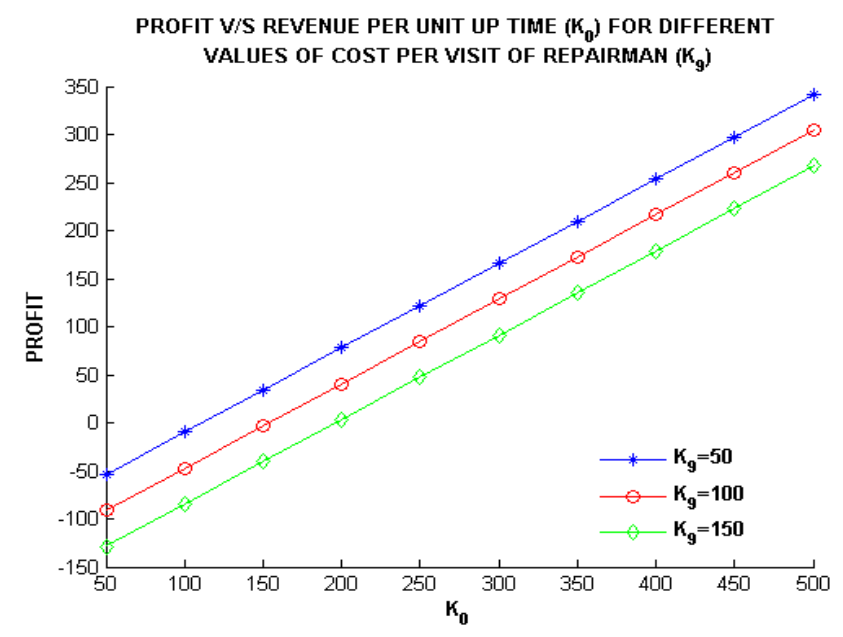

Figure 5.

Figure 5 shows the behavior of profit function with respect to revenue per unit up time $\left(K_{0}\right)$ for different values of cost per visit of the repairman $\left(\mathrm{K}_{9}\right)$. It can be concluded from the graph that the profit get increases with the increase in values of $\mathrm{K}_{0}$ and has lower values for higher values of $K_{9}$. The values of other parameters are $\lambda_{2}=0.0002 / \mathrm{hr}, \lambda_{3}=0.00025 / \mathrm{hr}, \lambda_{4}=0.00019 / \mathrm{hr}, \lambda_{5}=$ $0.0039 / \mathrm{hr}, \quad \beta_{\mathrm{a} 1}=3 / \mathrm{hr}, \quad \beta_{\mathrm{a} 2}=3.5 / \mathrm{hr}, \quad \beta_{\mathrm{b} 1}=3.5 / \mathrm{hr}$, $\beta_{\mathrm{b} 2}=4 / \mathrm{hr}, \beta_{\mathrm{c}}=2 / \mathrm{hr}, \beta_{\mathrm{d}}=2.3 / \mathrm{hr}, \beta_{\mathrm{e}}=2 / \mathrm{hr}, \mathrm{p}_{1}=0.2, \mathrm{p}_{2}=0.4$, $\lambda_{1}=1 / \mathrm{hr}, \alpha_{4}=1.3 / \mathrm{hr}, \alpha_{5}=1.8 / \mathrm{hr}, \mathrm{p}_{2}=0.4, \mathrm{~K}_{1}=$ INR 400 , $\mathrm{K}_{2}=$ INR 600, $\mathrm{K}_{3}=$ INR 500, $\mathrm{K}_{4}=$ INR 300, $\mathrm{K}_{5}=$ INR 200, $\mathrm{K}_{6}=\mathrm{INR} 500, \mathrm{~K}_{7}=\mathrm{INR} 500, \mathrm{~K}_{8}=\mathrm{INR} 250$.

It is evident from the graph that

i. The profit increases with the increase in the rate of occurrence of revenue per unit up time when other parameters remain fixed.

ii. For $\mathrm{K}_{9}=50$, the profit is $>$ or $=$ or $<0$ according as $\mathrm{K}_{0}$ is $<$ or $=$ or $>103.223$. Hence the system is profitable to the plant whenever $\mathrm{K}_{0}>103.223$.

iii. For $K_{9}=100$, the profit is $>$ or $=$ or $<0$ according as $\mathrm{K}_{0}$ is $<$ or $=$ or $>167.837$. Hence the system is profitable to the plant whenever $\mathrm{K}_{0}>167.837$.

For $K_{9}=150$, the profit is $>$ or $=$ or $<0$ according as $K_{0}$ is $<$ or $=$ or $>201.024$. Hence the system is profitable to the plant whenever $\mathrm{K}_{0}>201.024$.

Figure 6 shows the graphs between profit and the rate of occurrence of faults in subsystem $\mathrm{D}\left(\lambda_{4}\right)$ for different values of repair rate $\left(\beta_{\mathrm{d}}\right)$. The graph conclude that the profit declined with increase in the values of rates of occurrence of faults in subsystem $\mathrm{D}$ and taking large values for large values of repair rate $\left(\beta_{\mathrm{d}}\right)$. The values of various other parameters are $\lambda_{2}=0.0002 / \mathrm{hr}$, $\lambda_{1}=0.00025 / \mathrm{hr}, \lambda_{5}=0.00019 / \mathrm{hr}, \lambda_{3}=0.0039 / \mathrm{hr}, \beta_{\mathrm{a} 1}=3 / \mathrm{hr}$, $\beta_{\mathrm{a} 2}=3.5 / \mathrm{hr}, \beta_{\mathrm{b} 1}=3.5 / \mathrm{hr}, \beta_{\mathrm{b} 2}=4 / \mathrm{hr}, \beta_{\mathrm{e}}=2.3 / \mathrm{hr}, \beta_{\mathrm{c}}=2 / \mathrm{hr}$, $\alpha_{3}=1 / \mathrm{hr}, \alpha_{4}=1.3 / \mathrm{hr}, \alpha_{5}=1.8 / \mathrm{hr}, \mathrm{p}_{1}=0.6, \mathrm{p}_{2}=0.4, \mathrm{~K}_{0}=\mathrm{INR}$ $1000, \mathrm{~K}_{1}=$ INR 400, $\mathrm{K}_{2}=$ INR 600, $\mathrm{K}_{3}=$ INR 500, $\mathrm{K}_{4}=\mathrm{INR} 300, \mathrm{~K}_{5}=\mathrm{INR} 200, \mathrm{~K}_{6}=\mathrm{INR} 500, \mathrm{~K}_{7}=\mathrm{INR}$ 500, $\mathrm{K}_{8}=$ INR 250, $\mathrm{K}_{9}=$ INR 650.

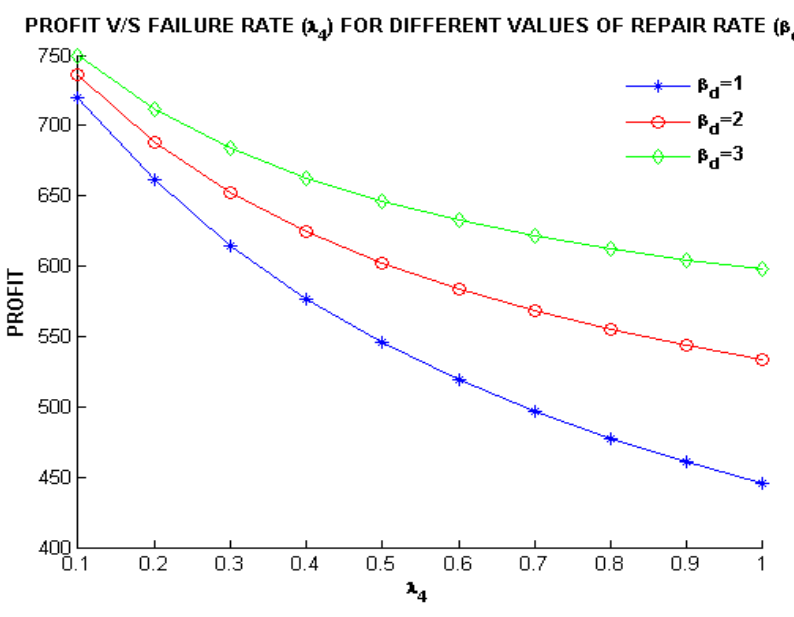

Figure 6.

\section{References}

[1] Kulshreshtha DK (1970). "Reliability of a repairable multicomponent system with redundancy in parallel”, IEEE Trans. Reliability 19, 50-52.

[2] EL-Said, K.M. and M.S. EL-Sherbeny (2010). "Stochastic Analysis of a Two-Unit Cold Standby System with Two-Stage Repair and Waiting Time”, Sankhya: the Indian journal of Statistics, 72(B), 1-10.

[3] Yusuf, B. and I. Yusuf (2013). "Evaluation Some Reliability Characteristics of a System under Three Types of Failures with Repair-Replacement at Failure”, American Journal of Operational Research, 3(3), 83-91.

[4] Taneja, G. and S. Sachdeva (2016). “Availability and Profit Analysis of Uni-directional and Revertible 1:1 Protection Scheme in Optical Communication Process”, International Journal of Pure and Applied Mathematics, 111 (3), 381-391.

[5] Rajesh, G. Taneja and P. Jagdish (2018). "Reliability of a Gas Turbine System with Change in Weather and Optimisation of Electricity Price when Working in Single Cycle”, Int. J. Agricult. Stat. Sci. 14(1), 119-128.

[6] Black, G. and Proschan, F. (1959). "On optimal redundancy”, Operations Research, 7(5), 581-588.

[7] Arora JR (1976). "Reliability of several standby priority redundant systems”, IEEE Trans. Reliability, 26, 290-293.

[8] Dhillon BS (1980). "Multistate device redundant system with common cause failures and one standby unit”, Microelectronics and Reliability 20(4), 411-417.

[9] Wang KH, Dong WL and Ke JB (2006). "Comparison of Reliability and Availability Between Four Systems with Warm Standby Components and Standby Switching Failure”, Applied Mathematics and Computation, 183, 1310-1322.

[10] Reena and Rajeev Kumar (2018). "Profit and Reliability Analysis of a Ceramic Tile Production System Considering Various Subsystem Failures”, International Journal of Applied Engineering Research, 13(10), 8436-8446. 